\title{
CONTROL AUTOMATION OF MARITIME UNMANNED COMPLEX WITH A GROUP OF AUTONOMOUS UNDERWATER VEHICLES
}

\author{
Volodymyr Blintsov \\ Department of electrical engineering of ship and robotic complexes \\ Admiral Makarov National University of Shipbuilding \\ 9 Heroiv Ukrainy ave., Mykolaiv, Ukraine, 54025 \\ volodymyr.blintsov@nuos.edu.ua \\ Leo Tosin Aloba \\ Department of electrical engineering of ship and robotic complexes \\ Admiral Makarov National University of Shipbuilding \\ 9 Heroiv Ukrainy ave., Mykolaiv, Ukraine, 54025 \\ alobat@gmail.com
}

\begin{abstract}
It is expedient to perform underwater search operations on large water areas using a group of autonomous self-propelled underwater vehicles. However, with a large distance to the search areas, the sea transition (from one point to the other) of the underwater vehicles requires high energy costs. This leads to the necessity to use heavy-duty underwater vehicles, which determines the high cost of the search operation. The transport of underwater vehicles is proposed to be carried out with an unmanned surface vessel, equipped with actuators for the automatic release of a group of vehicles under water and receiving on board after the end of the underwater mission. The maritime unmanned complex consisting of an unmanned surface vessel and a group of autonomous underwater vehicles on its board forms a new type of marine robotics, the complete automation of which is an actual scientific and technical task. For its implementation, the underlying (basic) automation technology of the marine search underwater mission has been developed as the theoretical basis for the development of the generalized structure of the complex automatic control system. Ten implementation stages of the underlying technology are formulated and the analysis of their automation features with the use of modern methods in the field of marine robotics is performed. Automation of the underlying technology stages involves the transfer of the vessel to a given water area, the automatic release (launch) of the group of underwater vehicles and their coordinated motion to the search area, the search operations and the return to the unmanned surface vessel, as well as the recovery of the vessel to the base. The generalized requirements for automatic control systems constituting the maritime unmanned complex at each stage of its functioning are provided. The spiral trajectory of waiting for the motion of the underwater vehicles at the group formation stages, for the search operation execution and after its completion, is proposed. For the spatial motion of the autonomous underwater vehicle as an agent of the group, the automatic control system was improved by introducing the blocks of the "Navigation Situation Model" and the "Navigation Threat Identifier, which make it impossible for emergency collision with the neighboring underwater vehicles of the group and disintegrate the group due to the data communication loss between them.
\end{abstract}

Keywords: maritime unmanned complex, autonomous underwater vehicle, underlying technology, automatic control.

DOI: $10.21303 / 2461-4262.2019 .00940$

\section{Introduction}

Autonomous Underwater Vehicle (AUV) is an effective instrument for carrying out a wide range of marine underwater search missions (exploratory) in nature $[1,2]$.

However, when performing such operations on large areas of water, the performance of a single AUV is low, which leads to an increase in the duration of underwater missions or even to the failure of their implementation.

One of the effective technologies for conducting unmanned maritime underwater missions is the group application of the AUVs, which guarantees the rapid survey of large areas with minimal time expenditure [3]. With such technology, underwater vehicles have hydroacoustic communication with each other, which ensures their collective operation and its high performance. 
However, the transition of the AUVs group to a given water area to carry out their search mission, as a rule, requires an increased resource of their on-board power supplies. This necessitates the use of high-energy AUVs, which increases the total cost of marine search operations.

It is expedient to carry out the delivery of the AUVs group to the search operation area using the unmanned surface vessel (USV). This will eliminate the need for each AUV to perform a long-distance maritime transition to a given search water area; will allow the use of AUV with relatively low energy supply, which is sufficient for maneuvering the USV and performing own underwater search mission.

Thus, to perform the maritime search mission, it is proposed to create a Maritime Unmanned Complex (MUC) as part of the USV and the AUVs group is located on its board.

The main problems of creating such a complex include the determination of its modes of operation and the development of a generalized automatic control system (ACS) of the complex in these modes. In particular, it is necessary to ensure the automatic transition of the MUC to a given point in the water area, the launch of the AUVs group into the sea, data communication with them and returning (recovery) to the USV board after the completion of the underwater search mission.

However, one of the important tasks of automatic control of the MUC underwater mission is to organize a coordinated spatial motion of the AUVs without the contact of neighboring vehicles and without excessively increasing the distance between them, which can cause the disappearance of the connection between the AUVs and the group breakup. Obviously, this task can be accomplished by improving the ACS of the individual AUVs on the account of obtaining additional information about the external navigation environment and the AUVs motion threats that exist in it.

\section{Literature review and problem statement}

The issue of group control of autonomous vehicles-robots has been highlighted over the past 10 years $[4,5]$. The works associated with the use of Multi-Agent Systems (MAS) in maritime environmental and archaeological researches are of particular relevance [6,7]. At present, the issues of control automation of a group of agents using complex onboard navigation devices and receivers of satellite navigation systems, radio channels, etc., have been rather fully investigated [8-10]. Such systems use multipurpose collaborative search algorithms, adaptive prediction, and wildlife-based algorithms (dolphins, fishes, etc.).

The researchers pay considerable attention to the mathematical modeling of the AUVs group motion processes, in particular, the creation of models of the external underwater environment and the identification of underwater threats, as well as the identification of the AUVs own parameters [11-13].

However, the issue of automation of robotic marine complexes, which ensure operative transportation of AUVs to remote areas of underwater search operations, has not been adequately investigated. In particular, the development of the automatic launch system, of the AUVs group in such water areas are required and their group operation until the full implementation of the search mission.

The aim of the article is to develop the basic tasks of the automatic control of a maritime unmanned complex, which performs the underwater search mission on a large remote maritime water area.

To achieve this goal, the following tasks are proposed to be solved:

- to develop an underlying technology for the automatic execution of marine underwater missions of searching nature with the help of the MUC and to determine its functioning features;

- to improve the ACS of the spatial motion of a individual AUV operating in the conditions of uncertainty of the external underwater environment characteristics by introducing the simulation blocks of external navigation environment and identification of navigation threats.

\section{The development results of MUC control automation tasks}

\section{1. Development of a basic production technology of marine underwater missions of} search nature using MUC

The modern experience of creating and using maritime robotics equipments $[14,15]$ allows defining the following ten basic stages of implementing the production underlying technology of maritime underwater search missions $A_{\mathrm{MUC}}$ : 
1 - MUC automatic transition $S C_{\mathrm{MUC1}}$ from the port of departure to the designated point of the sea area concerned, where the launch of the AUVs group is planned;

2 - automatic launch of the group from the USV board in the sea $R S_{\mathrm{AUV}}$ at a predetermined point of the sea area;

3 - self-organization (automatic formation) of the released AUVs to $F_{\text {MAS1 }}$ group for collective transition to the working zone, where the underwater mission will be performed;

4 - automatic group transition of the released AUVs $T_{\text {MASI }}$ to the working zone of the predetermined water area;

5 - automatic engagement by each AUV of the group of initial position $S_{\text {MAS }}$ for coordinated spatial motion in a predetermined search trajectory;

6 - AUVs group automatic implementation of the underwater search mission to the designated point $J_{\text {MAS }}$ (for example: search, identification and mapping of underwater objects);

7 - self-organization (automatic assembly) of AUVs into group $F_{\text {MAS2 }}$ for return to the USV;

8 - automatic group transition of AUVs to USV $T_{\text {MAS2 }}$;

9 - automatic return of the AUVs group aboard the USV $C S_{\mathrm{AUV}}$ :

10 - automatic transition of MUC $S C_{\mathrm{MUC} 2}$ to the home port.

Thus, the main stages of realizing the MUC basic task $A_{\text {MUC }}$ can be represented by a set:

$$
A_{\mathrm{MUC}}=\left\{S C_{\mathrm{MUCl}} ; R S_{\mathrm{AUV}} ; F_{\mathrm{MAS} 1} ; T_{\mathrm{MAS}} ; S_{\mathrm{MAS}} ; J_{\mathrm{MAS}} ; F_{\mathrm{MAS} 2}: T_{\mathrm{MAS} 2} ; C S_{\mathrm{AUV}} ; S C_{\mathrm{MUC} 2}\right\} .
$$

Let's now consider the main features of the organization of the MUC controlled motion during the maritime search operation.

The first and the tenth stages $\left(S C_{\mathrm{MUC1}} \in A_{\mathrm{MUC}} ; S C_{\mathrm{MUC} 2} \in A_{\mathrm{MUC}}\right)$ provide for the USV automatic motion with the AUVs group aboard from the port of departure to the scheduled point of release. Usually, such a transition occurs under conditions of external perturbations - wind and wave influences, flow, the appearance of navigational obstacles, etc. Therefore, the main requirements for the automatic control of USV motion are to ensure the safe motion of the vessel with a given trajectory with the estimated speed. At these stages of MUC mission, the known ACSs, created for the control of the USV can be used [16].

Automatic launch of the AUVs group from the USV board to the sea to the point with the predetermined geographic coordinates of the underwater search start ( $R S_{\mathrm{AUV}} \in A_{\mathrm{MUC}}$ second stage) and the automatic return of the AUVs group to the USV after the completion of the underwater search $\left(C S_{\mathrm{AUV}} \in A_{\mathrm{MUC}}\right.$ ninth stage) are complex scientific and technical tasks. Their solution is possible with the help of the vessel's actuating mechanism of the conveyor type, when for the release/return of the next AUVs from the USV board, a special underwater garage (UG) is lowered. At the same time, the USV is automatically positioned at the point of AUVs release ( $P_{\mathrm{USV}}$ mode).

The synthesis of such mechanisms and their control systems is a separate applied scientific and technical task and is not considered in this article.

The automatic formation of $A U V$ s released into the group, (third stage $F_{\mathrm{MAS} 1} \in A_{\mathrm{MUC}}$ ) for a collective transition to the working zone, where the underwater mission will be performed, forms a separate scientific task. The peculiarity of the automatic control of the search mission indicated stage is the need to move the AUVs along special trajectories of waiting. Such trajectories should provide for the content of the first released AUVs in the group in the process of releasing the next batch of underwater vehicles. It is advisable to first perform the release of the AUV-leader, which will form the trajectory of waiting.

When forming a plane (two-dimensional) AUVs group, such trajectories may take the form of an Archimedes spiral, diverging from the spiral pitch $a \leq r_{C}$, where $r_{C}$ is the range of the AUVs underwater communication system.

When forming a bulk (three-dimensional) AUVs group, the trajectory of waiting may take the form of several such spirals located at predetermined depths.

In the first case, when using the actuating mechanism of the conveyor type, the vessel's ACS, which controls the release of underwater vehicles, must carry out the release of AUVs with time interval: 


$$
\Delta t=s_{\mathrm{AUV}} / v_{\mathrm{AUV}},
$$

where $d<s_{\mathrm{AUV}}<r_{C}$ is the segment of the spiral trajectory of the released AUV; $\mathrm{d}$ is the minimum safe permissible distance between neighboring AUVs; $v_{\mathrm{AUV}}$ is the linear motion velocity of the released AUV.

When applying the AUV release mechanism of the cassette-type, the group of the underwater vehicles falls into water at the same time.

In the second case (the formation of the three-dimensional AUVs group), the vessel's ACS should begin to form the AUVs spiral trajectories of waiting from the highest given depth and complete the minimum predetermined depth.

In this case, the ACS of each released AUV must provide its plane motion along the Archimedes' spiral with a pitch $a \leq r_{C}$.

Group transition of the released $A U V S$. It is advisable to carry out a group transition of the released_AUVs to the working zone of a predetermined water area (the fourth stage $T_{\mathrm{MASl}} \in A_{\mathrm{MUC}}$ )) immediately after the release of the last group AUV. The beginning of the motion of each underwater vehicle in the group at a given depth of $H_{\mathrm{MAS} 1}$, given course $\varphi_{\mathrm{MAS} 1}$ and with a given speed $v_{\mathrm{MAS} 1}$ should be a common team, which is drawn from the USV ACS or from the AUV-leader [17]. One of the main tasks of the automation of this maritime search mission phase is AUVs coordinated motion without loss, i. e. without the collision of the neighboring underwater vehicles (vehicles excessive close approach) and without losing the connection (communication) between them (vehicles excessive distancing).

The automatic occupation of the initial position by each of the $A U V$ (fifth stage $\mathrm{S}_{\mathrm{MAS}} \in \mathrm{A}_{\mathrm{MUC}}$ ) for the coordinated spatial motion on the predetermined search trajectory is performed in accordance with the survey plan of a given water area, which has been pre-drawn up and entered into each AUV ACS. At the same time, lossless motion should be provided - avoiding AUVs collisions and removing beyond the limits of underwater communication systems operation.

The AUVS group automatically performs the underwater search mission (sixth stage $J_{\text {MAS }} \in A_{\text {MUC }}$ ) by the AUVs group coordinated motion to specified trajectories [18]. Navigation support for this mission stage can be organized using a bottom navigation system, pre-installed in the search area, or using navigation AUVs, equipped with GPS devices to determine own geographical coordinates, and hydroacoustic systems navigation support of underwater operations [19].

An important component of automatic control of this mission phase is also the AUVs group motion without collisions and loss of communication between the vehicles.

The AUVs automatic assembly into a group after the completion of the search operation to return to the USV (seventh stage $F_{\mathrm{MAS} 2} \in A_{\mathrm{MUC}}$ ) is performed by the AUV- leader's command. In the course of its implementation, the trajectory of waiting is formed by analogy with the third stage.

After collecting the full AUVs group, by the AUV-leader's command, starts the group transition to the USV ( $\left.T_{\mathrm{MAS} 2} \in A_{\mathrm{MUC}}\right)$. The motion of each underwater vehicle in the group at a given depth of $H_{\mathrm{MAS} 2}$, at a given course $\varphi_{\mathrm{MAS} 2}$ and at a given speed $v_{\mathrm{MAS} 2}$ should be without loss without collisions of neighboring vehicles and without loss of hydroacoustic communication between them.

The underlying technology for performing maritime underwater missions of a search nature with the help of MUC, taking into account the main modes of its operation, can be presented in the form of the algorithm shown in Fig. 1.

In Fig. 1 the marked symbols $Y 1 \ldots Y 10$, are the conditions for the stages implementation (1) of the implementation of the MUC basic task $A_{\text {MUC }}\left(x_{1}\right.$ is the planned progress of the implementation of the mission adequate phase; $x_{2}$ is the planned completion of the mission corresponding phase, $x_{3}$ is the unplanned stage progress, requiring access to the MUC ACS of higher level (transition B).

Other symbols on Fig. 1 correspond to (1).

The obtained algorithm can serve as the basis for the synthesis of the generalized structure of MUC ACS when performing underwater search missions. 


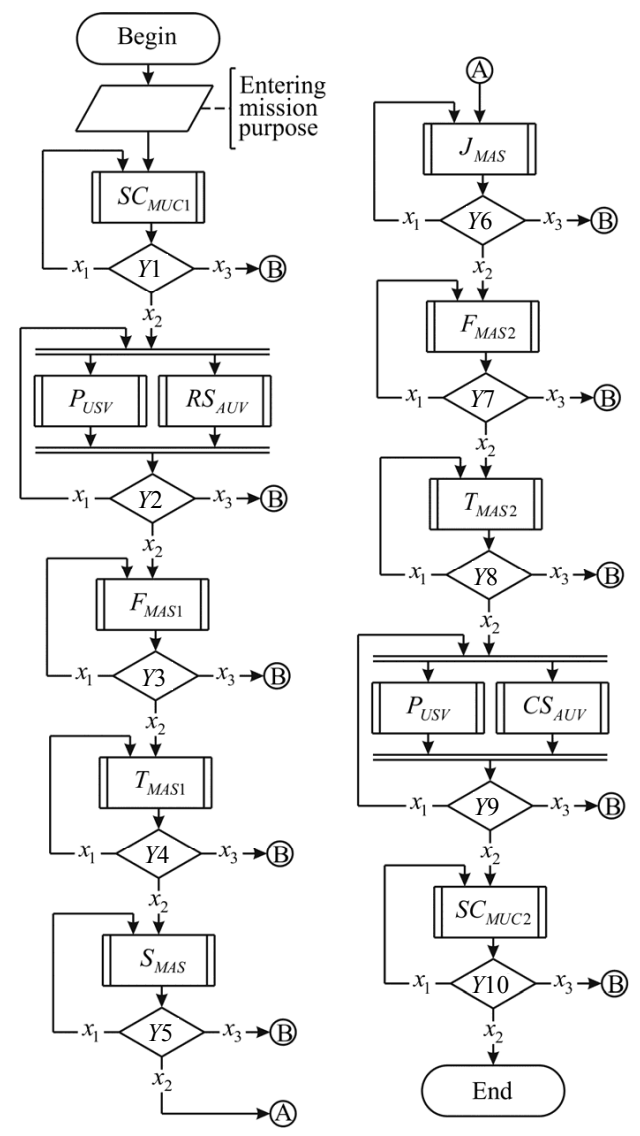

Fig. 1. The underlying technology of the search for maritime underwater missions using the MUC

\section{2. Synthesis of ACS of individual AUV spatial motion as a group agent}

Further, we will consider the plane motion of a single AUV as a group agent during the execution of the stages $T_{\mathrm{MAS} 1}$ and $T_{\mathrm{MAS} 2}$, as typical for organization of group motion in conditions of uncertainty in the characteristics of the external underwater environment. These uncertainties include the presence of neighboring AUVs that create navigation obstacles (in particular, the threats of collision during the group motion).

The AUVs group control tasks analysis at the specified stages of the maritime mission implementation indicates that one of the key tasks is ensuring the safe (trouble-free) motion of the individual AUVs in the group at a given depth of $H_{\mathrm{MAS}}$, at a given course $\varphi_{\mathrm{MAS}}$ and at a given speed $v_{\text {MAS }}$. The theoretical basis for the automation of such motion $A_{\mathrm{MUC}}$ is the notion of alignment $A_{G U}$, adhesion $A_{G A}$ and cohesion $A_{G C}$ [20].

The concept of alignment implies the observance of the above three mentioned parameters of

$$
A_{G U}=\left\{\varphi_{\mathrm{MAS}} ; v_{\mathrm{MAS}} ; H_{\mathrm{MAS}}\right\}
$$

which requires the AUVs group motion in a given direction or a given spatial trajectory and automatically control the motion of an individual AUV as a group agent in a given direction with the recommended given speed and at a given depth, which is the same for all the group AUVs.

The concept of adhesion involves the task of each AUV as a group agent to move with the consideration of $A_{G U}$ and simultaneously control the minimum possible distance between an individual agent and its neighbors, which is safe from a collision point of view:

$$
A_{G A}=\left\{x_{i} \geq\left. x_{\min }\right|_{i=1, \ldots S}\right\}
$$


where $x_{i}$ is the distance from its AUV to the neighboring AUV at the course angle $i ; x_{\min }$ is the minimum safe distance to the neighboring AUV; $S$ is the number of course angles controlled by the appropriate navigation range finders of its AUV.

The concept of cohesion involves the task of each group agent to move with regard to $A_{G U}$ and simultaneously controlling the maximum possible distance between an individual agent and its neighbors, safe from the point of view of loss of sensory contact (for example, hydroacoustic communication) and, consequently, the loss from the AUVs group as a result of its "dispersion":

$$
A_{G C}=\left\{x_{i} \leq\left. x_{\max }\right|_{i=1, \ldots S}\right\}
$$

where $x_{\max }$ is the maximum allowable distance between its AUV and the neighboring AUV, which maintains a reliable sensory contact between the vehicles.

Hydroacoustic, laser, or electrical sensors may be used as the AUV rangefinders, which should provide the underwater vehicle automatic control system sensitivity to neighboring AUVs moving in the group [21, 22].

The number of sensors and their directional patterns depend on a number of requirements in the AUVs group motion and on the performance peculiarities of the search underwater mission. Typically, the number of sensors varies from 4 to 12 and provides measurements of distances between the AUVs at the corresponding angular angles of the ranges from $0.1 \mathrm{~m}$ to $20 \mathrm{~m}$, which makes it possible to build efficient group motion control systems.

It is obvious that when performing the general requirement (3) in order to avoid collisions with the neighboring AUVs, our underwater vehicle has two main types of maneuvers - course maneuver and speed maneuver. Depth maneuver will be considered an emergency, undesirable in terms of the mission.

For the evaluation of the degree of collision threat between the AUVs group agents, it is suggested to include in the ACS for each AUV the special blocks - the "Navigation Situation Model (NSM)" block around the appropriate AUV and the "Navigation Threat Identifier (NTI)" block for this AUV. It is expedient to build these blocks using the theory of fuzzy logic [23]. Consider the purpose of these blocks in more detail.

The main idea of the NSM formation for a specific AUV is to formulate, for its AUV, the current underwater navigational situation with the definition of the nearest AUV-neighbors, which form the threat of collision from the controlled $S$ directions, and to determine the distances to them $X=\left\{x_{1} ; \ldots x_{S}\right\}$.

The task of the NTI block is to determine the dynamic parameters of the motion of the detected AUV-neighbors and to quantitatively calculate the level of danger of a collision or loss of contact with it (the characteristics of adhesion and cohesion of its AUV with respect to the nearest AUV-neighbors).

The generalized ACS structure of a single AUV as a group agent is shown in Fig. 2.

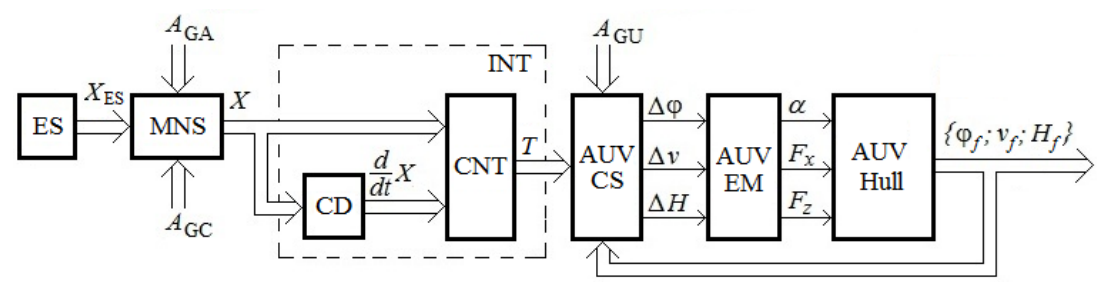

Fig. 2. Generalized ACS structure of a single AUV as a group agent

Consider the basic principles of operation of the above ACS.

The environment sensor (ES) block contains a set of $N$ group motion sensors $X_{E S}=$ $=\left\{X_{E S 1}, \ldots X_{E S i} ; \ldots X_{E S N}\right\}$ sensors with the targets which are shown in their measurement sectors). At the exit of the ES block, multiple sets of distances are formed to the neighboring AUV, and each set of signals $X_{E S i}$ may contain several distances, if there are several AUV-neighbors in the $i$-th sensor 
measurement sector. The course angles on such targets are determined by the arrangement of the corresponding sensor on the AUV hull in the linked coordinate system [24].

The $X_{E S}$ signal-based NSM block forms the targets current static picture around its AUV and defines $N$ closest AUV-neighbors that form the greatest threat of collision for it, and calculates the distances $X_{E S}=\left\{X_{E S 1} ; \ldots X_{E S i} ; \ldots X_{E S N}\right\}$.

The NTI threat Identification block evaluates the degree of collision threat with the AUV-neighbors and/or the threat of losing contact with the AUVs group in the event of a horizontal maneuver according to dependencies (4), (5), and also calculates a forecast of the group motion dynamics identified by AUV-neighbors. The NTI block contains a module for calculating the derivative distances of identified targets (Calculus of Derivative, CD) and navigational threats calculator (Calculus of Navigation Threat, CNT), which is proposed to be built on the basis of fuzzy logic [23]. The output signal of the NTI block is a recommendation vector for maneuvering in order to avoid threats $T=\left\{T_{1} ; \ldots ; T_{i} ; \ldots ; T_{N}\right\}$, where $T_{i}=f\left(\varphi_{i} ; v_{i} ; H_{i}\right)$ is a recommendation on the maneuver of its AUV to avoid collision or intolerable removal from the $i$-th AUV-neighbor.

The AUV automatic control system (AUV ACS) summarizes the calculated recommendations, using them as corrective signals for generating control actions $\{\Delta \varphi ; \Delta v ; \Delta H\}$ on the AUV actuating mechanisms (Executive Mechnism, EM) - the steering wheel is steered (the controlled value is the rudder angle $\alpha$ ), the marching propulsion device (the controlled value is the thrust on the longitudinal axis Fx) and, in emergency cases, its vertical propulsion device (the controlled value is the thrust on the vertical axis $F z$ ).

The aforementioned control influences applied to the AUV hull, cause the appearance of the actual variables of the spatial motion of our AUV $-\left\{\varphi_{f} ; v_{f} ; H_{f}\right\}$, which are used as feedback signals in the automatic control system of the underwater vehicle, AUV ACS.

\section{Discussion of the development of MUC control automation tasks}

Group application of AUV provides high performance underwater search operations. However, when it is necessary to perform an underwater search in remote areas, its performance is significantly reduced, since underwater vehicles have to travel long distances to move from the base to the place of work. Therefore, a maritime unmanned complex consisting of an unmanned surface vessel and the AUVs group on board is promising equipment for carrying out prospecting operations in such water areas.

The development of the automatic control system for unmanned surface vessels as the carrier of the AUVs group and the automatic control system for the AUVs group motion during the execution of the search mission are among the actual tasks of the MUC. For their synthesis, it is necessary to perform an analysis of the main stages of the MUC and determine the features of the AUVs group application, which are produced from the board of such a vessel.

The underlying technology proposed in the article for performing marine underwater missions of a search character with the help of an MUC forms the theoretical basis for the development of the generalized structure of the automatic control system of the MUC. It contains a description of the complete set of stages of the complex operation and analysis of the peculiarities of their automation, using modern advances in the field of marine robotics.

Based on the analysis of uncertainties that occurs during the AUVs group motion, the need to improve the automatic control system of a single AUV as an agent of the group has been shown. It was proposed to introduce into its structure the block "Navigation situation Model" and the block "Navigation threats Identifier", which will exclude the threat of an emergency collision of the groups neighboring AUVs and the disintegration of the group due to the loss of communication between the AUV. At the same time, the AUVs group motion is preserved by a given course at a given depth.

Cumulatively, the obtained theoretical results allow us to further formulate the tasks of automatic control systems synthesis for individual stages of the MUC and modes of operation of its executive mechanisms, as well as synthesis for control systems for safe spatial motion of an AUV as an agent of the group. 


\section{Conclusions}

To improve the performance of underwater search operations in remote marine areas, it was proposed to use an maritime unmanned complex as part of an unmanned surface vessel with a group of autonomous underwater vehicles on board. The relevance of the applied scientific task of control automation of such a complex is shown, as well as the need to improve the automatic control system of an individual AUV as an agent of the group, working in conditions of uncertainty.

An underlying technology has been developed for performing a marine underwater search mission with the help of the MUC as a carrier of a group of autonomous uninhabited underwater vehicles. It envisages ten stages, which generally cover the operations of automatic transition of the MUC to a given water area, automatic release of the AUVs group and their coordinated movement to the search area, performing search operations and returning to the unmanned surface vessel, as well as automatically returning the vessel to the base.

The peculiarities of the application of the MUC are considered and the generalized requirements for the automatic control systems of the components of the MUC at each stage of its operation are provided. In particular, the proposed spiral 'trajectory of waiting' for AUVs that are in the water, during the implementation of the formation stage of the AUVs group and the collection stage of the individual AUVs into the group after the search operation is completed.

The automatic control system of the spatial motion of an individual AUV, which performs the sea transition as part of a group and operates in conditions of uncertainty, has been developed. It was proposed to introduce into the control system the blocks "of the Navigation Situation Model" and the "Navigation Threats Identifier", which make it impossible for an emergency collision of the neighboring AUVs groups and the disintegration of the group due to the loss of information communication between the AUVs.

Further automation of the MUC with the AUVs group is seen in the control systems synthesis for executive mechanisms of unmanned surface vessels and in the synthesis of the blocks "Navigation Situation Model" and "Navigation Threats Identifier" of the AUV ACS.

\section{References}

[1] Button, R. W., Kamp, J., Curtin, T. B., Dryden, J. (2009). A Survey of Missions for Unmanned Undersea Vehicles. National Defense Research Institute. RAND Corporation, 223.

[2] Autonomous Vehicles in Support of Naval Operations (2005). Washington, DC: The National Academies Press, 256. doi: https:// doi.org/10.17226/11379

[3] Chatzichristofis, S. A., Kapoutsis, A., Kosmatopoulos, E. B., Doitsidis, L., R., D., Borges de Sousa, J. (2017). The NOPTILUS project: Autonomous Multi-AUV Navigation for Exploration of Unknown Environments. IFAC (International Federation of Automatic Control). Available at: http://www.openarchivescy.com/Record/hephaestus-11728-10204/ Description\#tabnav

[4] Yasuda, T. (Ed.) (2011). Multi-Robot Systems, Trends and Development. Published by InTech, 586. doi: http://doi.org/10.5772/544

[5] Tsiogkas, N., Papadimitriou, G., Saigol, Z., Lane, D. (2014). Efficient multi-AUV cooperation using semantic knowledge representation for underwater archaeology missions. 2014 Oceans - St. John's. doi: https://doi.org/10.1109/oceans.2014.7003085

[6] Li, Z., Duan, Z. (2017). Cooperative control of multi-agent systems: A consensus region approach. CRC Press, 262. doi: https:// doi.org/10.1201/b17571

[7] Li, J., Zhang, J., Zhang, G., Zhang, B. (2018). An Adaptive Prediction Target Search Algorithm for Multi-AUVs in an Unknown 3D Environment. Sensors, 18 (11), 3853. doi: https://doi.org/10.3390/s18113853

[8] Sotzing, C. C., Evans, J., Lane, D. M. (2007). A Multi-Agent Architecture to Increase Coordination Efficiency in Multi-AUV Operations. OCEANS 2007 - Europe. doi: https://doi.org/10.1109/oceanse.2007.4302393

[9] Das, B., Subudhi, B., Pati, B. B. (2016). Co-operative control of a team of autonomous underwater vehicles in an obstacle-rich environment. Journal of Marine Engineering \& Technology, 15 (3), 135-151. doi: https://doi.org/10.1080/20464177 .2016 .1247636

[10] Hai, H., Guocheng, Z., Hongde, Q., Zexing, Z. (2017). Autonomous underwater vehicle precise motion control for target following with model uncertainty. International Journal of Advanced Robotic Systems, 14 (4), 172988141771980. doi: https:// doi.org/10.1177/1729881417719808 
[11] Mousavian, S. H., Koofigar, H. R. (2016). Identification-Based Robust Motion Control of an AUV: Optimized by Particle Swarm Optimization Algorithm. Journal of Intelligent \& Robotic Systems, 85 (2), 331-352. doi: https://doi.org/10.1007/ s10846-016-0401-9

[12] Tolba, S., Ammar, R., Rajasekaran, S. (2015). Taking swarms to the field: A framework for underwater mission planning. 2015 IEEE Symposium on Computers and Communication (ISCC). doi: https://doi.org/10.1109/iscc.2015.7405645

[13] Vanhée, L., Borit, M., Santos, J. (2018). Autonomous Fishing Vessels Roving the Seas: What Multiagent Systems Have Got to Do with It. AAMAS '18 Proceedings of the 17th International Conference on Autonomous Agents and MultiAgent Systems, 1193-1197. Available at: http://ifaamas.org/Proceedings/aamas2018/pdfs/p1193.pdf

[14] Guo, W., Wang, S., Dun, W. (2015). The Design of a Control System for an Unmanned Surface Vehicle. The Open Automation and Control Systems Journal, 7 (1), 150-156. doi: https://doi.org/10.2174/1874444301507010150

[15] Willcox, S., Goldberg, D., Vaganay, J., Curcio, J. A. Multi-vehicle cooperative navigation and autonomy with the bluefin cadre system. Available at: https://www.researchgate.net/publication/241654294_multi-vehicle_cooperative_navigation_and_ autonomy_with_the_bluefin_cadre_system

[16] Blintsov, V. S., Aloba, L. T., Tkhy, D. F. (2016). Modern problems of group motion control of remotely operated underwater vehicles. Zbirnyk naukovykh prats NUK, 3 (465), 83-91.

[17] Matsuda, T., Maki, T., Sato, Y., Sakamaki, T., Ura, T. (2017). Alternating landmark navigation of multiple AUVs for wide seafloor survey: Field experiment and performance verification. Journal of Field Robotics, 35 (3), 359-395. doi: https://doi.org/ 10.1002/rob.21742

[18] Santos, V. G., Chaimowicz, L. (2014). Cohesion and segregation in swarm navigation. Robotica, 32 (2), 209-223. doi: https:// doi.org/10.1017/s0263574714000563

[19] $1 \mathrm{MHz} / 200 \mathrm{KHz}$ Dualfrequency Underwater Depth Measurement Ultrasonic Transducer. Available at: http://www.chinaseniorsupplier.com/Electronic_Components_Supplies/Active_Components/747348205/1MHz_200KHz_Dual_frequency_Underwater_Depth_Measurement_Ultrasonic_Transducer.html

[20] Miskovic, N., Vukic, Z., Petrovic, I., Barisic, M. (2009). Distance keeping for underwater vehicles - tuning Kalman filters using self-oscillations. OCEANS 2009-EUROPE. doi: https://doi.org/10.1109/oceanse.2009.5278139

[21] Burunina, Z. Yu., Voitasyk, A. M., Aloba, L. T., Korytskyi, V. I., Sirivchuk, A. S., Klochkov, A. P. (2018). Experimental Study of Group Control Laws for an Autonomous Unmanned Underwater Vehicle as a Group Agent. Shipbuilding and Marine Infrastruture, 2 (10), 116-126.

[22] Blintsov, O. (2017). Devising a method for maintaining manageability at multidimensional automated control of tethered underwater vehicle. Eastern-European Journal of Enterprise Technologies, 1 (9 (85)), 4-16. doi: https://doi.org/10.15587/17294061.2017.93291

Received date 16.06.2019

Accepted date 01.07.2019

Published date 31.07.2019
(C) The Author(s) 2019

This is an open access article under the CC BY license (http://creativecommons.org/licenses/by/4.0). 